\title{
ASYMPTOTIC BEHAVIOR OF PERTURBED AUTONOMOUS LINEAR FUNCTIONAL DIFFERENTIAL EQUATIONS
}

\author{
RICHARD B. EVANS
}

\begin{abstract}
It is shown that certain autonomous linear functional differential equations and their perturbations satisfy a generalized type of asymptotic equivalence. An example is given.
\end{abstract}

1. Introduction. The purpose of this paper is to study the comparative asymptotic behavior of certain linear functional differential equations and their perturbations. The case when both equations are ordinary has been studied extensively by such authors as Brauer and Wong [2] and Hallam and Hiedel [6]. If the unperturbed equation is an ordinary differential equation, but the perturbed equation is not, the theory of ordinary differential equations may still be relied upon as in Hallam, Ladas and Lakshmikantham [7], and Talpalaru [12].

The behavior of solutions to functional differential equations can be considerably different from that of solutions of ordinary differential equations and hence it is worthwhile to consider the case where the linear equation need not be ordinary. This problem has been studied by Kato [9], [10], Hale [5], Cooke [3], and Lakshmikantham and Leela [11]; however these studies consider the case when the solutions are bounded or they require other restrictions not imposed here.

The methods employed below involve the variation of constants formula, the comparison principle, and the Schauder-Tychonoff theorem. The proofs are analogous to those given for ordinary differential equations. Due to the added difficulty encountered with dependence on past time, it is convenient to require that the linear equation be autonomous. The results rely on the theory found in Hale [4]. The notation is al so that of Hale [4].

Let $\sigma \in R, r \geq 0$, and $C=C\left([-r, 0], R^{n}\right)$. Consider the autonomous linear functional differential equation

Received by the editors June 18, 1973 and, in revised form, December 19, 1973. AMS (MOS) subject classifications (1970). Primary 34K25.

Key words and phrases. Asymptotic behavior, perturbed autonomous linear functional differential equations, variation of constants, Schauder-Tychonoff theorem, generalized asymptotic equivalence. 


$$
\dot{u}(t)=L\left(u_{t}\right)
$$

with $u_{\sigma}=\phi \in C$, and the perturbed equation

$$
\dot{x}(t)=L\left(x_{t}\right)+F\left(t, x_{t}\right)
$$

with $x_{\sigma}=\psi \in C$. For $\phi \in C$ suppose that

$$
L(\phi)=\int_{-r}^{0} d \eta(\theta) \phi(\theta)
$$

where $\eta$ is an $n \times n$ matrix function of bounded variation on $[-r, 0]$. Further assume that the measure induced by $\eta$ contains no singular part. Let $F: R \times C \rightarrow R^{n}$ be continuous and map closed bounded sets into bounded sets.

The functions $L$ and $F$ will be required to satisfy further hypotheses so that a scalar comparison technique can be applied. To this end the following is introduced. Let $\omega(t, \lambda)$ be a continuous nonnegative function on $[\sigma, \infty) \times R^{+}$which is increasing in $\lambda$ and which is such that for some $M>$ 0 the maximal solution $r(t)$ of

$$
\dot{r}(t)=\omega(t, r(t)), \quad r(\sigma)=M,
$$

exists for $t \geq \sigma$ and satisfies $\lim _{t \rightarrow \infty} r(t)=r_{\infty}<\infty$.

2. Main results. Theorem 1 below states that corresponding to some solutions of (2) there exist solutions of (1) which satisfy a certain asymptotic relationship (see (3) below). Theorem 2 considers the converse problem.

One of the techniques (see Brauer and Wong [2]) used to compare solutions of ordinary differential equations with solutions of perturbations of these systems has been to use the variation of constants formula and impose hypotheses so that a family of solutions $u(t)$ of the unperturbed system can be continued for $t \rightarrow-\infty$. This technique must be modified for functional differential equations since only a few solutions can be continued for $t \rightarrow-\infty$. That is, for $\Lambda$ a set of eigenvalues of (1), and $C$ decomposed by $\Lambda$ as $C=P \oplus Q, T(t) \phi^{P}$ can be continued for $t \rightarrow-\infty$ and $\phi \in C$.

Theorem 1. Let $\Lambda$ be a finite set of eigenvalues of (1), and let $C$ be decomposed by $\Lambda$ as $C=P \oplus Q$. Let $D(t)$ be a continuous $n \times n$ nonsingular matrix for $t \geq \sigma-r$. For $\phi \in C$, suppose $L$ and $F$ satisfy:

(i) $\left|D_{t} T(t-\sigma) \phi\right| \leq K|\phi|, t \geq \sigma$,

(ii) $\left|D_{t} T(t-s) X_{0}^{P} F(s, \phi)\right| \leq 1 / 2 \omega\left(s,\left|D_{s} \phi\right|\right), \sigma \leq t, \sigma \leq s$,

(iii) $\left|D_{t} T(t-s) X_{0}^{Q} F(s, \phi)\right| \leq 1 / 2 e^{-\gamma(t-s)} \omega\left(s,\left|D_{s} \phi\right|\right), \sigma \leq s \leq t$, where $K$, $y>0$. Then for $\phi \in C$ and $|\phi|$ small, there exists a solution $x(t)$ of (2) for 
$t \geq \sigma$ with $x_{\sigma}=\phi$. To each such solution there corresponds a solution $u(t)$ of (1) for $t \geq \sigma$ such that

$$
\lim _{t \rightarrow \infty}\left|D_{t}\left(x_{t}-u_{t}\right)\right|=0 .
$$

Proof. Choose $\phi \in C$ such that $K|\phi|<M$. Let $x(t)$ be a solution of (2) with $x_{\sigma}=\phi$. From the variation of constants formula and (i)-(iii), we obtain

$$
\left|D_{t} x_{t}\right| \leq M+\int_{\sigma}^{t} \omega\left(s,\left|D_{s} x_{s}\right|\right) d s
$$

which implies that (see Hartman [8])

$$
\left|D_{t} x_{t}\right| \leq r(t)
$$

as long as $x(t)$ exists. The inequality (4) and Hale [4, Theorem 4.2] imply that $x(t)$ is defined and satisfies (2) for $t \geq \sigma$.

The condition (ii) and (4) can be used to show that the integral

$$
\int_{\sigma}^{\infty} D_{. t}(\theta) T(t-s) X_{0}^{P}(\theta) F\left(s, x_{s}\right) d s
$$

exists uniformly for $t \geq \sigma$ and $\theta \in[-r, 0]$; and since $D(t)$ is nonsingular it follows that

$$
\int_{\sigma}^{\infty} T(t-s) X_{0}^{P}(\theta) F\left(s, x_{s}\right) d s
$$

exists uniformly for $\theta \in[-r, 0]$ and $t$ in compact subintervals of $[\sigma, \infty)$. The decomposition of the variation of constants formula (see Hale [4]) takes the form

$$
x_{t}=z(t)-\int_{t}^{\infty} T(t-s) X_{0}^{P} F\left(s, x_{s}\right) d s+\int_{\sigma}^{t} T(t-s) X_{0}^{Q} F\left(s, x_{s}\right) d s,
$$

where

$$
z(t)=T(t-\sigma) \phi+\int_{\sigma}^{\infty} T(t-s) X_{0}^{P} F\left(s, x_{s}\right) d s
$$

Easy calculations show that $u(t)$ defined by

$$
u(t)= \begin{cases}z(t)(0), & t \geq \sigma, \\ z(\sigma)(t-\sigma), & \sigma-r \leq t \leq \sigma,\end{cases}
$$

satisfies $u_{t}=z(t)$ and is a solution of (1). Finally using (ii), (iii), and (4) we have

$$
\left|D_{t}\left(x_{t}-u_{t}\right)\right| \leq \int_{\sigma}^{t} e^{-\gamma(t-s)} \omega(s, r(s)) d s+\int_{t}^{\infty} \omega(s, r(s)) d s .
$$

An exercise in Brauer and Nohel [1, p. 182] implies that (3) holds. 
The next theorem will be a partial converse of Theorem 1, but it is convenient to state first the following lemma.

Lemma 1. Let the hypotheses in Theorem 1 be satisfied. Let $v:[\sigma, \infty)$ $\rightarrow C$ be a continuous function such that $\sup _{\sigma \leq t}\left|D_{t} \nu(t)\right|<r_{\infty}$. Then the in. tegral

$$
\int_{\sigma}^{\infty} T(t-s) X_{0}^{P}(\theta) F(s, v(s)) d s
$$

exists uniformly for $t$ in compact subintervals of $[\sigma, \infty)$ and $\theta \in[-r, 0]$. For $z:[\sigma, \infty) \rightarrow C$ defined by

$$
z(t)=\int_{\sigma}^{t} T(t-s) X_{0} F(s, v(s)) d s-\int_{\sigma}^{\infty} T(t-s) X_{0}^{P} F(s, v(s)) d s
$$

and $x:[\sigma-r, \infty) \rightarrow R^{n}$ defined by the right side of (5), we have $x_{t}=z(t)$ and

$$
\dot{x}(t)=L\left(x_{t}\right)+F(t, v(t)), \quad x_{\sigma}=-\int_{\sigma}^{\infty} T(\sigma-s) X_{0}^{P} F(s, v(s)) d s .
$$

Finally $x$ satisfies

$$
x_{t}=\int_{\sigma}^{t} T(t-s) X_{0}^{Q} F(s, v(s)) d s-\int_{t}^{\infty} T(t-s) X_{0}^{P} F(s, \nu(s)) d s .
$$

Proof. The first conclusion is obtained as in the proof of Theorem 1. The second and third conclusions are verified by straightforward calculations.

Theorem 2. Let the hypotheses of Theorem 1 hold. Let $u(t)$ be a solution of (1) for $t \geq \sigma$ with $u_{\sigma}=\phi \in C$. If $|\phi|$ is sufficiently small, there exists $a \tau \geq \sigma$ and a solution $x(t)$ of (2) for $t \geq \tau$ such that (3) holds.

Proof. In the following we will construct a set $H$ and a mapping $S$ on $H$ and apply the Schauder-Tychonoff theorem to obtain a fixed point. This method was used by Hallam and Hiedel [6] for ordinary differential equations.

Suppose $\phi \in C$ and $K|\phi|<r_{\infty}$. Let $\rho=1 / 2\left(r_{\infty}-K|\phi|\right)$ and pick $\tau \geq \sigma$ such that

$$
\int_{\tau}^{t} e^{-\gamma(t-s)} \omega\left(s, r_{\infty}-\rho\right) d s+\int_{t}^{\infty} \omega\left(s, r_{\infty}-\rho\right) d s \leq 2 \rho
$$

for $t \geq \tau$. Let $J=[\tau-r, \infty)$ and $C_{\tau}=C\left(J, R^{n}\right)$ with the topology of uniform convergence on compact subintervals of $J$.

The set

$$
H=\left\{z \in C_{\tau}: z(t)=D(t) x(t) \text { for } x \in C_{\tau} \text { and }\left|z_{t}\right| \leq r_{\infty}-\rho \text { for } t \geq \tau\right\}
$$

is a closed convex subset of $C_{\tau}$. Let $S: H \rightarrow C_{\tau}$ be defined by 


$$
(S z)_{t}=D_{t}\left\{u_{t}+\int_{\tau}^{t} T(t-s) X_{0}^{Q} F\left(s, D_{s}^{-1} z_{s}\right) d s-\int_{t}^{\infty} T(t-s) X_{0}^{P} F\left(s, D_{s}^{-1} z_{s}\right) d s\right\}
$$

for $t \geq \tau$ where $D_{t}^{-1}=\left(D^{-1}\right)_{t}$. Lemma 1 shows that this defines a mapping from $H$ into $C_{\tau}$ and, using (i)-(iii) and (6), we get $\left|\left(S_{z}\right)_{t}\right| \leq r_{\infty}-\rho$ for $t \geq$ $\tau$ and, hence, $S(H) \subseteq H$.

Let $\left\{z^{n}\right\}_{n=1}^{\infty}$ be a sequence in $C_{\tau}$ which converges to $z$. Sets of the form

$$
\left\{\left(t, z_{t}^{n}\right): t \in\left[\tau, t_{1}\right], n=1,2, \cdots\right\} \cup\left\{\left(t, z_{t}\right): t \in\left[\tau, t_{1}\right]\right\},
$$

where $\tau \leq t_{1}<\infty$ are compact subsets of $\left[\tau, t_{1}\right] \times C_{\tau}$. Using the uniform continuity of $F$ on compact sets, it can be shown that $S z^{n}$ converges to $S z$. Hence $S$ is continuous.

Because $S(H) \subseteq H$, the functions in $S(H)$ are uniformly bounded. It remains only to show that they are equicontinuous on compact subintervals of $J$.

Define the family of functions $G$ by

$$
G=\left\{y \in C_{\tau}: y_{t}=D_{t}^{-1}(S z), z \in H, t \geq \tau\right\} .
$$

Lemma 1 implies that if $y_{t}=D_{t}^{-1}(S z)$, then $y$ is a solution of

$$
\begin{aligned}
\dot{y}(t) & =L\left(y_{t}\right)+F\left(t, D_{t}^{-1} z_{t}\right), \quad t \geq \tau, \\
y_{\tau} & =u_{\tau}-\int_{\tau}^{\infty} T(\tau-s) X_{0}^{P} F\left(s, D_{s}^{-1} z_{s}\right) d s .
\end{aligned}
$$

The terms $L\left(D_{t}^{-1}(S z)_{t}\right)$ and $F\left(t, D_{t}^{-1} z_{t}\right)$ are uniformly bounded for $z \in H$ and $t$ in compact subintervals of $[\tau, \infty)$. Also the integral in $y_{\tau}(\theta)$ converges uniformly for $\theta \in[-r, 0]$ and $z \in H$. Hence the family $G$ is equicontinuous when restricted to compact subintervals of $\mathrm{J}$.

For $t$ and $t^{\prime}$ in a compact subinterval of $J$, the inequality

$$
\left|S z(t)-S z\left(t^{\prime}\right)\right| \leq|D(t)|\left|y(t)-y\left(t^{\prime}\right)\right|+\left|D(t)-D\left(t^{\prime}\right)\right|\left|y\left(t^{\prime}\right)\right|
$$

shows that $S(H)$ is equicontinuous on compact subintervals of $J$. Hence the closure of $S(H)$ is compact and by the Schauder-Tychonoff theorem, $S$ has a fixed point $z$ in $H$.

We have seen that $x(t)=D^{-1}(t) S z(t)=D^{-1}(t) z(t)$ satisfies

$$
\begin{aligned}
\dot{x}(t) & =L\left(x_{t}\right)+F\left(t, x_{t}\right), \quad t \geq \tau, \\
x_{\tau} & =u_{\tau}-\int_{\tau}^{\infty} T(\tau-s) X_{0}^{P} F\left(s, x_{s}\right) d s,
\end{aligned}
$$

which is (2). As in the proof of Theorem 1 we find that (3) holds. 
3. An example. The following example (see Hale [5]) will be an application of Theorems 1 and 2. Suppose $r=n=1$ and $\sigma=0$. Let $L$ be defined by $L(\phi)=\phi(0)-\phi(-1)$. Then (1) has the form $\dot{u}(t)=u(t)-u(t-1)$. Take $\Lambda$ $=\{0\}$. The functions $\phi_{1}(\theta)=1$ and $\phi_{2}(\theta)=\theta$, with $-1 \leq \theta \leq 0$, form a basis for $P$. By Hale [4] there exist $\gamma>0$ and $K>0$ such that

$$
\begin{aligned}
& \left|T(t) \phi^{Q}\right| \leq 1 / 2 K e^{-\gamma t}\left|\phi^{Q}\right|, \quad t \geq 0, \\
& \left|T(t) X_{0}^{Q}\right| \leq 1 / 2 K e^{-\gamma t}, \quad t \geq 0 .
\end{aligned}
$$

Define $F: R \times C \rightarrow R$ by

$$
F(t, \phi)=a_{1}(t) \phi^{2}(0)-\alpha_{2}(t) \phi^{2}(-1),
$$

where $a_{i}: R \rightarrow R, i=1$ and 2 , are continuous and $\int_{0}^{\infty}(s+2)^{3} \alpha(s) d s<\infty$ for $\alpha(t)=\left|\alpha_{1}(t)\right|+\left|\alpha_{2}(t)\right|$. Equation (2) becomes

$$
\dot{x}(t)=\left[1+\alpha_{1}(t) x(t)\right] x(t)+\left[1-\alpha_{2}(t) x(t-1)\right] x(t-1) .
$$

We can choose $M>0$ such that $M K \int_{0}^{\infty}(s+2)^{3} \alpha(s) d s<1$ for $K>0$. Then

$$
r(t)=M\left[1-M K \int_{0}^{t}(s+2)^{3} \alpha(s) d s\right]^{-1}
$$

is the unique bounded solution to

$$
r(t)=K(t+2)^{3} a(t) r^{2}(t), \quad r(0)=M .
$$

If we take $D(t)=1 /(t+2)$ on $-1 \leq t$, and $\omega(t, \lambda)=K(t+2)^{3} \alpha(t) \lambda^{2}$, the hypotheses of Theorems 1 and 2 are satisfied.

\section{REFERENCES}

1. F. Brauer and J. A. Nohel, The qualitative theory of ordinary differential equations, Benjamin, New York, 1969.

2. F. Brauer and J. S. W. Wong, On asymptotic behavior of perturbed linear systems, J. Differential Equations 6 (1969), 142-153. MR 39 \#570.

3. K. L. Cooke, Linear functional differential equations of asymptotically autonomous type, J. Differential Equations 7 (1970), 154-174. MR 41 \#604.

4. J. Hale, Functional differential equations, Springer-Verlag, New York, 1971.

5. - Linear asymptotically autonomous functional differential equations, Rend. Circ. Mat. Palermo. (2) 15 (1966), 331-351. MR 38 \#1367.

6. T. G. Hall am and J. W. Hiedel, The asymptotic manifolds of a perturbed linear system of differential equations, Trans. Amer. Math. Soc. 149 (1970), 233241. MR $41 \# 2136$.

7. T. G. Hallam, G. Ladas and V. Lakshmikantham, $O n$ the asymptotic behavior of functional differential equations, SIAM J. Math. Anal. 3 (1972), 58-64. MR $47 \# 3796$. 
8. P. Hartman, Ordinary differential equations, Wiley, New York, 1964. MR 30 $\# 1270$.

9. J. Kato, On the existence of a solution approaching zero for functional differential equations, Proc. U. S.-Japan Seminar on Differential and Functional Equations (Minneapolis, Minn., 1967), Benjamin, New York, 1967, pp. 153-169. MR 37 $\# 1740$.

10. - The asymptotic equivalence of systems of functional differential equations, J. Differential Equations 1 (1965), 306-332. MR 31 \#1485.

11. V. Lakshmikantham and S. Leela, Differential and integral inequalities, theory and applications, Vol. II, Academic Press, New York, 1969.

12. Pavel Talpalaru, Quelques problèmes concernant l'équivalence asymptotique des systèmes différentiels, Bol. Un. Mat. Ital. (4) 4 (1971), 164-186. MR 45\#7201.

DEPARTMENT OF MATHEMATICS, LOUISIANA STATE UNIVERSITY, NEW ORLEANS, LOUISIAN A 70122 